\title{
Morbus Adamantiades-Behçet: erfolgreiche Therapie mit ungewöhnlichen Komplikationen
}

\author{
Ioannis Karagiannidis, Christos C. Zouboulis
}

\begin{abstract}
Orale Aphthen, Genitalulzera und Uveitis oder Iritis - das ist die klassische Trias des Morbus Adamantiades-Behçet. Die seltene chronische Krankheit verläuft schubförmig, und das klinische Bild ist sehr variabel. Nicht nur die Diagnose kann schwierig sein: Dieser Fall zeigt, dass auch Nebenwirkungen der Therapie den Verlauf prägen können.
\end{abstract}

\begin{abstract}
Anamnese
Die 37-jährige Patientin türkischer Herkunft kam bereits unter dem Verdacht auf einen Morbus Adamantiades-Behçet in unsere Klinik. Eine orale Aphthose war seit dem 16. Lebensjahr bekannt, insbesondere an den Lippen und im Rachenbereich, mit monatlichen Schüben. Als Kind war sie an Asthma bronchiale und Meningitis erkrankt. Vor etwa 5 Jahren traten rezidivierend Erythemata nodosa auf, zunächst an beiden Sprunggelenken und anschließend an den Unterschenkeln, begleitet von Schmerzen der Hand- und Fußgelenke.
\end{abstract}

Vor 3 Monaten fanden sich erstmals Genitalulzera; zeitgleich trat eine Panuveitis auf. Die Patientin erhielt vom Augenarzt $20 \mathrm{mg} / \mathrm{d}$ Prednisolon oral, ohne dass sich die Befunde wesentlich besserten. Zur Therapieoptimierung wurde sie in unser spezialisiertes Zentrum überwiesen.

\section{Verlauf unter Therapie}

Die Zusammenschau der Befunde $(\vee$ Tab. 1$)$ bestätigte den Verdacht eines Morbus AdamantiadesBehçet. Zur Behandlung eines gleichzeitig zur stationären Aufnahme aufgetretenen zweiten Uveitis-Schubs mit Papillenödem, Vaskulitis, Vitritis sowie Netzhautblutung erhielt die Patientin zunächst über 3 Monate $400 \mathrm{mg} / \mathrm{d}$ Ciclosporin A oral.

Tab. 1 Untersuchungsbefunde bei der Erstaufnahme.

- Darauf kam es zu einer ausgeprägten Hypertrichose.

\footnotetext{
Körperliche Gewicht $68 \mathrm{~kg}$, Größe $155 \mathrm{~cm}$

Untersuchung Körpertemperatur: $36,5^{\circ} \mathrm{C}$

Druckdolentes rechtes Handgelenk, rechtes Knie und rechte Fußwurzel

2 Aphthen am Rachen

Genitalulzera

Laborbefunde

Leukozyten 12,9Gpt/I (Norm: 4,8-10,8)

Segmentkernige Neutrophile 9,93Gpt/l (Norm: 2,40-7,56)

C-reaktives Protein 10 mg/I (Norm: 0-5)

Pathergietest

Negativ

Bildgebung

MRT des Schädels, Skelettszintigraphie, EEG: ohne pathologischen Befund

Funduskopie

Rechter Fundus: Blutung am oberen Gefäßbogen
}

Wir stellten die Therapie auf $2 \times 50 \mathrm{mg} / \mathrm{d}$ Azathioprin und $30 \mathrm{mg} / \mathrm{d}$ Prednisolon oral um.

- Trotzdem entwickelte sich 4 Monate später eine Thrombophlebitis im rechten Oberschenkel.

Aufgrund der persistierenden Aktivität der Erkrankung und der Augensymptomatik mit Erblindungsgefahr erhielt die Patientin Interferon- $\alpha-2 a$ (IFN $\alpha-2 a)$. Die Dosis wurde innerhalb von 6 Tagen von 1,5 auf $3 \times 9$ Mio IE pro Woche s.c. erhöht. Da Interferon in Kombination mit Azathioprin zu Myelosuppression führen kann, setzten wir Azathioprin ab. Unter IFN $\alpha-2 a$ gingen die oralen und genitalen Ulzera zurück, und vor allem die okulären Befunde besserten sich deutlich.

- Innerhalb von 2 Wochen klagte die Patientin jedoch über Müdigkeit und Angstzustände - häufige Nebenwirkungen von IFN $\alpha$-2a.

Wir klärten die Patientin über den Zusammenhang der IFN $\alpha$-2a-Therapie mit Depressionen auf. Da sie die erstmalige deutliche Besserung des schweren Augenbefalls nicht gefährden wollte,

- behandelten wir die Depression mit Citalopram $20 \mathrm{mg} / \mathrm{d}$, Lorazepam $4 \times 0,5 \mathrm{mg} / \mathrm{d}$ und Promethazin $20 \mathrm{mg} / \mathrm{d}$ zur Nacht, jeweils oral

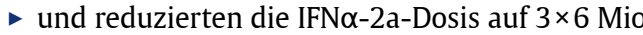
IE/Woche subkutan.

Als weitere Nebenwirkung der IFN $\alpha$-2a-Therapie kam es nach 3 Monaten zu diffusem Haarausfall. Nach 14 Monaten traten eine Mastodynie und Hyperprolaktinämie auf $(57,1 \mathrm{ng} / \mathrm{ml}$; Normbereich $4,8-$ 23,3). Die gynäkologische Untersuchung ergab keinen weiteren pathologischen Befund. Eine Kernspintomographie der Sella schloss ein Prolaktinom aus. Die Schilddrüsenwerte waren ebenfalls unauffällig.

- Die Patientin erhielt Bromocriptin 2,5 mg/d.

Zwei Monate nach Reduktion der IFN $\alpha$-2a-Dosis wurde sie ungeplant schwanger (es handelte sich um ihre vierte Schwangerschaft)

- Außer IFN $\alpha-2 a$ ( $3 \times 6$ Mio IE/Woche) wurden alle Medikamente pausiert.

- Wegen Panikattacken erhielt die Patientin das trizyklische Antidepressivum Doxepin, $50 \mathrm{mg} / \mathrm{d}$ zur Nacht. 
Inzwischen, 2 Jahre nach der Entbindung eines gesunden Kindes, bestehen noch verstärkte Episoden mit Kopfschmerzen und Abgeschlagenheit. Prolaktin ist weiterhin erhöht, mit gleichzeitiger Galaktorrhoe links, trotz erneuter Gabe von Bromocriptin $2 \times 2,5 \mathrm{mg} / \mathrm{d}$. Wiederholte kernspintomographische Untersuchungen gaben nach wie vor keine Hinweise auf ein Prolaktinom. Der Morbus Adamantiades-Behçet ist unter IFN $\alpha-2 a$ in Remission geblieben - bis auf das gelegentliche Auftreten einzelner oraler Aphthen.

\section{Diskussion}

Der Morbus Adamantiades-Behçet ist weltweit verbreitet, tritt aber vor allem im östlichen Mittelmeerraum sowie im Mittleren und Fernen Osten endemisch auf $[1,16]$. Sehr gut wirksam, insbesondere im Hinblick auf die okulären Manifestationen der Erkrankung, ist die Therapie mit IFN $\alpha$-2a [3, 4, $6-8,14,15]$. Sie hat jedoch vielfältige, gelegentlich schwere Nebenwirkungen, v.a.

- grippeähnliche Symptome, Müdigkeit, Kopfschmerzen, Gelenkschmerzen,

- Leukopenie und Thrombopenie $[11,14]$.

Auch Depressionen (bis zu Suizidalität) und reversibler diffuser Haarausfall kommen vor. Bei unserer Patientin trat kurz nach Therapiebeginn eine klinisch relevante Depression auf. Sie verschlimmerte sich nach 3 Jahren während einer Schwangerschaft und wird seitdem medikamentös behandelt.

Die Datenlage zum Morbus Adamantiades-Behçet bei Schwangeren ist uneinheitlich: Es finden sich

- sowohl vermehrt schwere Komplikationen

- als auch milde Krankheitsverläufe.

- Insbesondere ist das Thromboserisiko erhöht. In Fallberichten aus der Türkei und Israel besserten sich die Symptome während der Schwangerschaft, es traten jedoch auch Komplikationen, insbesondere Gefäßmanifestationen und Thrombosen, bei Schwangeren mit Morbus Adamantiades-Behçet auf [12].Zudem zeigte eine Auswertung von 27 Schwangerschaften aus Korea mehrheitlich eine Verschlechterung des Krankheitsverlaufes [2]. Eine spanische Studie ergab keine erhöhte Komplikationsrate [9].

Ausreichende Erfahrungen mit IFN $\alpha$-2a bei Schwangeren liegen nicht vor. Tierversuche erbrachten keine Hinweise auf teratogene Wirkungen. Bei unserer Patientin war die Wahrscheinlichkeit sehr hoch, dass es ohne IFN $\alpha$-2a zu einem Rezidiv der im Vorfeld therapierefraktären Uveitis gekommen wäre. Daher erhielt sie das Medikament auf ihren Wunsch und nach Rücksprache mit dem betreuenden Gynäkologen weiterhin, allerdings in abgeschwächter Dosis. Während der Schwangerschaft wurde die Patientin besonders engmaschig kontrolliert.

Veröffentlichungen über eine Hyperprolaktinämie unter IFN $\alpha$-2a gibt es unseres Wissens bisher nicht.

\section{Medikamente}

Angiotensin II

Antiarrhythmika: Verapamil

Antidepressiva: MAO-Hemmer, SSRI, trizyklische

Antidepressiva

Antiemetika: Domperidon, Metoclopramid

Antihistaminika

Antihypertensiva

Hormone

Opioide, endogene Opiate

indirekte Dopaminantagonisten: Naltrexon

Neuroleptika: Phenothiazine

Die Wirkung von Interferon auf die Prolaktinsekretion ist umstritten:

- Nach Hofland et al. [5] hemmt IFN $\alpha-2 a$ die Prolaktinsekretion bei kultivierten humanen hypophysären Adenomen.

- Dagegen zeigten Yamaguchi et al. [13], dass die Interferon-Familie die Prolaktinsekretion in vitro stimuliert.

Diese Beobachtungen genügen jedoch nicht, um eine Korrelation zwischen einer symptomatischen Hyperprolaktinämie und der Behandlung mit IFN $\alpha$ 2a sicher auszuschließen.

Eine chronische Hyperprolaktinämie kann nicht nur durch ein Prolaktinom, sondern auch durch Hypothyreose, Niereninsuffizienz, Stress, Gravidität und Medikamente ( $\bullet$ Tab. 2) ausgelöst werden. Bei unserer Patientin zeigten wiederholte Kernspintomographien kein Prolaktinom. Auch die Schilddrüsenparameter waren wiederholt unauffällig. Die Hyperprolaktinämie kann auch der Gravidität nicht zugeschrieben werden, da sie bereits viele Monate vor Schwangerschaftsbeginn festgestellt wurde.

- Einerseits könnten Antidepressiva und Neuroleptika die Hyperprolaktinämie ausgelöst haben.

- Andererseits könnte auch die IFN $\alpha-2 a-T h e r a p i e$ die Hyperprolaktinämie hervorgerufen haben.

So hatten Patienten mit multipler Sklerose unter IFN- $\beta$-Therapie eine Hyperprolaktinämie [10]. IFN- $\alpha$ und $-\beta$ belegen den gleichen Rezeptor - sie weisen therapeutisch ähnliche, aber nicht identische Wirkungen und Nebenwirkungen auf $[11,14]$.

Konsequenz für Klinik und Praxis

- IFNa-2a ist beim Morbus Adamantiades-Behçet sehr gut wirksam.

- Die Therapie ist jedoch mit langwierigen Nebenwirkungen verbunden.

- Mastodynie und Hyperprolaktinämie als mögliche Nebenwirkungen von IFNa-2a werden hier unseres Wissens zum ersten Mal beschrieben.

- Allerdings könnte bei unserer Patientin auch die Einnahme von Antidepressiva für diese Nebenwirkungen eine Rolle gespielt haben.
Tab. 2 Medikamente, die eine Hyperprolaktinämie auslösen können [17].

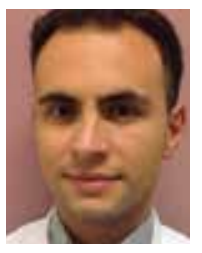

loannis Karagiannidis arbeitet als Assistenzarzt an der Klinik für Dermatologie, Venerologie und Allergologie/ Immunologisches Zentrum am Städtischen Klinikum Dessau

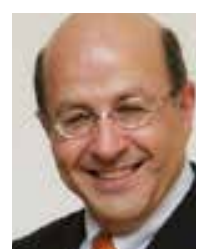

Prof. Dr. med. Dr. h.c. Christos C. Zouboulis leitet als Chefarzt die Klinik für Dermatologie, Venerologie und Allergologie / Immunologisches Zentrum am Städtischen Klinikum Dessau christos.zouboulis@klinikumdessau.de

Vollständiges Literaturverzeichnis unter

http://dx.doi.org/10.1055/ s-0040-100438

\section{Interessenkonflikte}

Die Autoren erklären, dass keine Interessenkonflikte bestehen.

DOI 10.1055/s-0040-100438 Dtsch Med Wochenschr 2015; 140: 112-113

(c) Georg Thieme Verlag KG . Stuttgart · New York .

ISSN 0012-0472 


\section{Literatur}

1 Altenburg A, Mahr A, Maldini C et al. Epidemiologie und Klinik des Morbus Adamantiades-Behçet in Deutschland. Ophthalmologe 2012; 109: 531-541

2 Bang D, Chun YS, Haam IB, Lee ES, Lee S. The influence of pregnancy on Behçet's disease. Yonsei Med J 1997; 38: 437-443

3 Bonitsis NG, Altenburg A, Krause L, Stache T, Zouboulis CC. Current concepts in the treatment of Adamantiades-Behçet's disease. Drugs Fut 2009; 34: 749-763

4 Deuter CM, Zierhut M, Möhle A, Vonthein R, Stöbiger $\mathrm{N}$, Kötter I. Long-term remission after cessation of interferon- $\alpha$ treatment in patients with severe uveitis due to Behçet's disease. Arthritis Rheum 2010; 62: 2796-2805

5 Hofland LJ, de Herder WW, Waaijers M et al. Interferonalpha-2a is a potent inhibitor of hormone secretion by cultured human pituitary adenomas. J Clin Endocrinol Metab 1999; 84: 3336-3343

6 Kötter I, Eckstein AK, Stübiger N, Zierhut M. Treatment of ocular symptoms of Behçet's disease with interferon alpha 2a: a pilot study. Br J Ophthalmol 1998; 82: 488-494

7 Kötter I, Zierhut M, Eckstein AK et al. Human recombinant interferon alfa-2a for the treatment of Behçet's disease with sight threatening posterior or panuveitis. Br J Ophthalmol 2003; 87: 423-431

8 Krause L, Altenburg A, Pleyer U, Köhler AK, Zouboulis $\mathrm{CC}$, Foerster $\mathrm{MH}$. Long-term visual prognosis of ocular Adamantiades-Behçet's disease treated with Interferonalfa-2a. J Rheumatol 2008; 35: 896-903

9 Marsal S, Falgá C, Simeon CP, Vilardell M, Bosch JA. Behçet's disease and pregnancy relationship study. $\mathrm{Br}$ J Rheumatol. 1997; 36: 234-238
10 Nabavi SM, Koupai SA, Nejati MR, Garshasbi E, Jalali MR. Menstrual irregularities and related plasma hormone levels in multiple sclerosis patients treated with beta interferon. Acta Med Iran 2010; 48: 36-41

11 Stübiger N, Winterhalter S, Pleyer U, Doycheva D, Zierhut M, Deuter $C$. Janus-faced? Effects and side-effects of interferon therapy in ophthalmology. Ophthalmologe 2011; 108: 204-212

12 Uzun S, Alpsoy E, Durdu M, Akman A. The clinical course of Behçet's disease in pregnancy: a retrospective analysis and review of the literature. J Dermatol. 2003; 30: 499-502

13 Yamaguchi M, Koike K, Matsuzaki N, Yoshimoto Y, Taniguchi T, Miyake A, Tanizawa O. The interferon family stimulates the secretions of prolactin and interleukin- 6 by the pituitary gland in vitro. J Endocrinol Invest. 1991; 14: 457-461

14 Zouboulis CC, Orfanos CE. Treatment of AdamantiadesBehçet's disease with systemic interferon alfa. Arch Dermatol 1998; 134: 1010-1016

15 Zouboulis CC, Treudler R, Orfanos CE. Morbus Adamantiades-Behçet: therapeutischer Einsatz von systemischem rekombinanten Interferon-alpha-2a. Hautarzt 1993; 44: 440-445

16 Zouboulis CC. Epidemiology of Adamantiades-Behçet's Disease. In: Zierhut M, Ohno S, editors. Immunology of Behçet's Disease. Lisse: Swets \& Zeitlinger; 2003. 1-16

17 Melmed S, Casanueva F. Hoffman A et al. Diagnosis and treatment of hyperprolactinemia: An Endocrine Society clinical practice guideline. J Clin Endocrinol Metab 2011; 96: 273-288 